\title{
A Changing Weights Spatial Forecast Combination Approach with an Application to Housing Price Prediction
}

\author{
Chuanhua Wei ${ }^{1}$, Chenping $\mathrm{Du}^{2} \&$ Nana Zheng ${ }^{1}$ \\ ${ }^{1}$ School of Science, Minzu University of China, Beijing, China \\ ${ }^{2}$ School of Mathematics, Sun Yat-Sen University, Guangzhou, China \\ Correspondence: Chenping Du, School of Mathematics, Sun Yat-sen University, Guangzhou 510220, China. Tel: \\ 86-178-2706-5504. E-mail: dcp9765@126.com
}

Received: January 31, 2020

Accepted: February 25, 2020

Online Published: March 5, 2020

doi:10.5539/ijef.v12n4p11

URL: https://doi.org/10.5539/ijef.v12n4p11

\begin{abstract}
Forecast combination has been widely applied in various fields since the seminal article of Bates and Granger (1969). However, these research were focused only on time series data. Few study focus on the spatial data, this paper proposes a novel adaptive spatial forecast combination method with varying weights based on the geographically weighted regression technique. Finally, the proposed method is applied to the Boston house prices prediction, and the results indicate that our procedure performs better than the other forecast combination methods.
\end{abstract}

Keywords: geographically weighted regression, combining forecast, local weighted least-squares approach, varying weights

\section{Introduction}

Forecast combination method has been theoretical studied in econometrics and applied in many fields for a long time since the seminal article of Bates and Granger (1969). The recent advances can be found in Granger (2006), Timmermann (2006), Claeskens et al. (2016) and Chan and Pauwels (2018).

As we all known, forecast combination method was focused only on time series forecasting problem. However, different to time series forecasting, spatial forecasting or spatial prediction is also very important in many areas, such as economics, geography, population studies, environment science, ecology and epidemiology. Spatial prediction methods can be divided into two classes, one is geostatistics (spatial statistics) methods, the other is spatial econometrics methods. In geostatistics, the prediction of unknown quantities at given locations is commonly made by the kriging technique. In addition to the kriging technique for modeling regular lattice spatial data, the spatial econometrics models including spatial autoregressive models and geographically weighted regression can also be used. More discussion and comparison on these two kinds of spatial prediction can be found in Tsutsumi and Seya (2009) and Mojiri et al. (2017). However, little efforts have been devoted to spatial forecast combination. This paper wants to fill up this gap, and proposes a novel spatial combining prediction method with varying weights based on the geographically weighted regression technique.

The rest of this paper is organized as follows. In Section 2, the spatial forecast combination method is proposed. In Section 3, the proposed approach is applied to the Boston house prices prediction. Conclusion is presented in Section 4.

\section{Method}

To proposed the new method, we briefly describe a simple combined forecast method based on the Ordinary Least Squares (OLS) regression. OLS-based forecast combination method was studied by Granger and Ramanathan (1984). They proposed to combine forecasts with unrestricted regression coefficients as weights.

Let $\mathrm{f}_{\mathrm{i}}^{\mathrm{j}}$ denote an out-of-sample prediction value of the variable $\mathrm{y}_{\mathrm{i}}$ by the $\mathrm{jth}$ individual forecasting approach at spatial location $\left(\mathrm{u}_{\mathrm{i}}, \mathrm{v}_{\mathrm{i}}\right)$, for $\mathrm{i}=1,2, \ldots, \mathrm{n}$ and $\mathrm{j}=1,2, \ldots, \mathrm{p}$. Then, we can define the following linear regression model.

$$
\mathrm{y}_{i}=\beta_{0}+\beta_{1} f_{i}^{1}+\cdots+\beta_{p} f_{i}^{p}+\varepsilon_{i}, i=1,2, \cdots, n
$$

Apply the Ordinary Least Squares approach to linear regression model (1), we can obtain the OLS estimator for 
regression coefficient $\beta=\left(\hat{\beta}_{0}, \hat{\beta}_{1}, \cdots, \hat{\beta}_{p}\right)^{T}$ as

$$
\hat{\beta}=\left(\hat{\beta}_{0}, \hat{\beta}_{1}, \cdots, \hat{\beta}_{p}\right)^{T}=\left(F^{T} F\right)^{-1} F^{T} Y,
$$

with

$$
F=\left|\begin{array}{lllll}
1 & \mathrm{f}_{1}^{1} & \mathrm{f}_{1}^{2} & \ldots & \mathrm{f}_{1}^{p} \\
1 & \mathrm{f}_{2}^{1} & \mathrm{f}_{2}^{2} & \cdots & \mathrm{f}_{2}^{p} \\
\vdots & \vdots & \vdots & \ddots & \vdots \\
1 & \mathrm{f}_{n}^{1} & \mathrm{f}_{n}^{2} & \cdots & \mathrm{f}_{n}^{p}
\end{array}\right|, Y=\left[\begin{array}{c}
y_{1} \\
y_{2} \\
\vdots \\
y_{n}
\end{array}\right] .
$$

Then, we can construct prediction value for $\mathrm{y}_{0}$ at a new location $\left(\mathrm{u}_{0}, \mathrm{v}_{0}\right)$ by the OLS combined forecast method. Firstly, we obtain prediction $\mathrm{f}_{0}^{\mathrm{j}}, \mathrm{j}=1,2, \ldots, \mathrm{p}$ by $\mathrm{p}$ individual forecasting methods. Then, combined forecast for $\mathrm{y}_{0}$ can be defined as

$$
\hat{\mathbf{y}}_{0}^{\mathrm{OLS}}=\hat{\boldsymbol{\beta}}_{0}+\hat{\beta}_{0} f_{0}^{1}+\cdots+\hat{\beta}_{p} f_{0}^{p} .
$$

To improve the constant weights forecast combination methods, Deutsch et al. (1994) proposed a time-varying weights combined forecast method. They considered the combination of forecasts using changing weights derived from switching regression models or from smooth transition regression models. Discussions on time-varying weights forecast combination method can be found in Billio et al. (2000), Terui and van Dijk (2002), Guidolin and Timmermann (2009) and Raftery et al. (2010). We are motivated to propose a spatial combined forecast method with spatial-varying weights.

Based on model (1), we can get the following spatial varying coefficient model by allowing the regression coefficients $\beta=\left(\hat{\beta}_{0}, \hat{\beta}_{1}, \cdots, \hat{\beta}_{p}\right)^{T}$ to change with spatial location,

$$
\mathrm{y}_{i}=\beta_{0}\left(\mathrm{u}_{i}, v_{i}\right)+\beta_{1}\left(\mathrm{u}_{i}, v_{i}\right) f_{i}^{1}+\cdots+\beta_{p}\left(\mathrm{u}_{i}, v_{i}\right) f_{i}^{p}+\varepsilon_{i}, i=1,2, \cdots, n
$$

Compared with linear model $(1), \beta(\cdot)=\left[\beta_{0}(\cdot), \beta_{1}(\cdot), \ldots, \beta_{\mathrm{p}}(\cdot)\right]^{\mathrm{T}}$ is not a p-dimensional vector of constants but unknown functions of geographical locations,

To estimate spatial varying model (4), Brunsdon et al. (1996) proposed a geographically weighted regression (GWR) approach. The GWR method estimates the coefficients by the local weighted least-squares technique in the framework of kernel smoothing. More references on GWR can be found in the monograph Fotheringham et al. (2002). Now, for a spatial location $\left(\mathrm{u}_{0}, \mathrm{v}_{0}\right)$, we apply this technique to estimate the regression coefficients $\beta\left(u_{0}, v_{0}\right)=\left(\hat{\beta}_{0}\left(u_{0}, v_{0}\right), \hat{\beta}_{1}\left(u_{0}, v_{0}\right), \cdots, \hat{\beta}_{p}\left(u_{0}, v_{0}\right)\right)^{T}$. Let $\mathrm{d}_{0 \mathrm{i}}$ be the distance between $\left(\mathrm{u}_{0}, \mathrm{v}_{0}\right)$ and the ith designed location $\left(\mathrm{u}_{\mathrm{i}}, \mathrm{v}_{\mathrm{i}}\right)$. The GWR estimation of $\beta\left(\mathrm{u}_{0}, \mathrm{v}_{0}\right)$ is to minimize the objective function

$$
\sum_{i=1}^{n}\left[\mathrm{y}_{i}-\beta_{0}\left(\mathrm{u}_{0}, v_{0}\right)-\sum_{\mathrm{j}=1}^{p} \beta_{j}\left(\mathrm{u}_{0}, v_{0}\right) f_{i}^{j}\right]^{2} K_{h}\left(d_{0 i}\right)
$$

and $\mathrm{K}$ is a kernel function, $\mathrm{h}$ is a bandwidth and $\mathrm{K}_{\mathrm{h}}(\cdot)=\mathrm{K}(\cdot / \mathrm{h}) / \mathrm{h}$.

Let $W\left(u_{0}, v_{0}\right)=\operatorname{diag}\left[K_{h}\left(d_{01}\right), K_{h}\left(d_{02}\right), \cdots, K_{h}\left(d_{0 n}\right)\right]$, and the solution to the weighted least-squares problem (5) can be explicitly expressed by

$$
\hat{\beta}\left(\mathrm{u}_{0}, v_{0}\right)=\left(\hat{\beta}_{0}\left(\mathrm{u}_{0}, v_{0}\right), \hat{\beta}_{1}\left(\mathrm{u}_{0}, v_{0}\right), \cdots, \hat{\beta}_{p}\left(\mathrm{u}_{0}, v_{0}\right)\right)^{T}=\left(F^{T} W\left(\mathrm{u}_{0}, v_{0}\right) F\right)^{-1} F^{T} W\left(\mathrm{u}_{0}, v_{0}\right) Y .
$$

As usual, the weight function $\mathrm{K}_{\mathrm{h}}(\cdot)$ in equation (5) can be chosen to be the Gaussian weight

$$
K_{h}\left(d_{0 i}\right)=\frac{1}{\sqrt{2 \pi}} \exp \left[-\frac{1}{2}\left(\frac{d_{0 i}}{h}\right)^{2}\right], i=1,2, \cdots, n,
$$

For the choice of bandwidth $\mathrm{h}$, various data-driven methods, such as cross-validation (CV), generalized cross-validation (GCV), and AIC, can be applied, more details can be found in Fotheringham et al. (2002). CV method will be employed in the next section.

Then, the GWR combined forecast for $\mathrm{y}_{0}$ based on individual forecasting values $f_{0}^{1}, f_{0}^{2}, \cdots, f_{0}^{p}$ can be defined as

$$
\hat{\mathbf{y}}_{0}^{\mathrm{GWR}}=\hat{\beta}_{0}\left(\mathbf{u}_{0}, v_{0}\right)+\hat{\beta}_{1}\left(\mathbf{u}_{0}, v_{0}\right) f_{0}^{1}+\cdots+\hat{\beta}_{p}\left(\mathbf{u}_{0}, v_{0}\right) f_{0}^{p}
$$

\section{Results}

We now illustrate the proposed estimating procedure by analyzing the Boston Housing data set, which was used originally by Harrison and Rubinfeld (1978) and corrected for a few minor errors by Gilley and Pace (1996). The 
data set consists of the median value of owner-occupied homes in 506 census tracts in the Boston Standard Metropolitan Statistical Area in 1970, along with 13 accompanying sociodemographic and related variables, and is now freely available through the Sedep package of the open source software R. Details of variables can be found in Table 1.

Table 1. Description of the Boston dataset

\begin{tabular}{|c|c|c|}
\hline Variable & Description & Defined variable \\
\hline MEDV & median value of owner-occupied homes in 1,000 USD & $Y=\ln (M E D V)$ \\
\hline CRIM & the per capita crime rate by town & $X_{1}=C R I M$ \\
\hline $\mathrm{ZN}$ & proportion of area zoned with large lots & $X_{2}=Z N$ \\
\hline INDUS & the proportion of non-retail business acres per town & $X_{3}=I N D U S$ \\
\hline CHAS & the Charles River dummy variable & $X_{4}=$ CHAS \\
\hline NOX & the nitrogen oxide concentration in parts per 10 million & $X_{5}=N O X^{2}$ \\
\hline $\mathrm{RM}$ & the average number of rooms in owner-occupied homes per dwelling & $X_{6}=R M^{2}$ \\
\hline AGE & the proportion of owner-occupied homes built prior to 1940 & $X_{7}=A G E$ \\
\hline DIS & the weighted distances to five employment centers in the Boston region & $X_{8}=\ln (D I S)$ \\
\hline RAD & the index of accessibility to radial highways & $X_{9}=\ln (R A D)$ \\
\hline TAX & the full value property tax rate per $10,000 \mathrm{USD}$ & $X_{10}=T A X$ \\
\hline PTRATIO & the pupil-teacher ratio by town school district & $X_{11}=$ PTRATIO \\
\hline B & the black proportion of population by town & $X_{12}=B$ \\
\hline LSTAT & the proportion of population that is in the lower status & $X_{13}=\ln (L S T A T)$ \\
\hline
\end{tabular}

We apply the following five models as individual forecasting procedures. $\mathrm{M}_{1}$ is a traditional linear regression model. To capture the "large-scale" locational effects between response variable and associated covariates, Pace and Gilley (1997) considered $\mathrm{M}_{2}$ with $\left(\mathrm{u}_{\mathrm{i}}, \mathrm{v}_{\mathrm{i}}\right)$ is the latitude (LAT) and longitude (LON) of the ith observation. $\mathrm{M}_{3}$ is a spatial error model, $\mathrm{M}_{3}$ is a spatial autoregressive model, and $\mathrm{M}_{4}$ is a spatial Durbin model. We use the Euclidean distance in terms of longitude and latitude to set up the spatial weight matrix $\mathbf{W}$. We choose the threshold distance to be 1 which gives a $\mathbf{W}_{\mathbf{n}}$ matrix with $1.18 \%$ nonzero elements.

$$
\begin{aligned}
& M_{1}: \quad Y_{i}=\beta_{0}+\sum_{j=1}^{13} \beta_{j} X_{i j}+\varepsilon_{i}, \\
& M_{2}: \quad Y_{i}=\beta_{0}+\sum_{j=1}^{13} \beta_{j} X_{i j}+\beta_{14} u_{i}+\beta_{15} v_{i}+\beta_{16} u_{i} v_{i}+\beta_{17} u_{i}^{2}+\beta_{18} v_{i}^{2}+\varepsilon_{i}, \\
& M_{3}: \quad Y_{i}=\beta_{0}+\sum_{j=1}^{13} \beta_{j} X_{i j}+\varepsilon_{i}, \varepsilon_{i}=\sum_{j=1}^{n} W_{i j} \varepsilon_{j}+e_{i}, \\
& M_{4}: \quad Y_{i}=\beta_{0}+\sum_{j=1}^{n} W_{i j} Y_{j}+\sum_{j=1}^{13} \beta_{j} X_{i j}+\varepsilon_{i}, \\
& M_{5}: \quad Y_{i}=\beta_{0}+\sum_{j=1}^{n} W_{i j} Y_{j}+\sum_{j=1}^{13} \beta_{j} X_{i j}+\sum_{j=1}^{13} \theta_{j} W_{i j} X_{i j}+\varepsilon_{i} .
\end{aligned}
$$

For 506 observations, we divided the data into a training set (486 observations) and a test set (20 observations). Based on the training set and the above five individual models $\mathrm{M}_{1}-\mathrm{M}_{5}$, we can obtain out-of-sample forecasting value for response variabe $\mathrm{Y}$ at each spatial location. Then, based on the true values of $\mathrm{Y}$ and the according four forecasting values, we can apply the above five combined forecast approaches to get weights of each individual forecasting method. Nextly, based on the training set and the above five individual models $\mathbf{M}_{1}-\mathrm{M}_{4}$, we can obtain individual forecasting values for response variabe $\mathrm{Y}$ of test set. Finally, we get combined forecasting values by their individual forecasting values and weights.

Based on the above five individual forecasting models, besides the proposed GWR-based forecast combinations method, we will apply the following four frequently used forecast combinations schemes. It is obviously that the four methods are all constant weights procedures. The proposed GWR method is a varying weights procedure. In our real data analysis, the weights for the individual forecasting models $M_{1}$ to $M_{5}$ according to these fours forecast combinations methods are computed by the R package ForecastCombinations. Denote $\mathrm{f}_{0}{ }^{\mathrm{k}}, \mathrm{k}=1,2, \ldots, 5$ 
are out-of-sample individual forecasting values for housing price at location $\left(\mathrm{u}_{0}, \mathrm{v}_{0}\right)$ according to individual models $\mathrm{M}_{1}$ to $\mathrm{M}_{5}$.

1) Simple average (SA). The most natural approach to combine forecasts is using the mean of all those forecasts. The combined forecast is defined as

$$
\hat{\mathrm{y}}_{0}^{\mathrm{SA}}=\frac{1}{5} \sum_{k=1}^{p} f_{0}^{k}
$$

2) OLS. The OLS-based combine forecasts method was introduced in Section 2, and the combined forecast is given as

$$
\hat{y}_{0}^{\text {OLS }}=-0.0907-0.7492 f_{0}^{1}+0.2953 f_{0}^{2}-0.0716 f_{0}^{3}+1.3072 f_{0}^{4}+0.2487 f_{0}^{5}
$$

3) Constrained Least Squares (CLS) regression. For linear model (1), OLS estimator was obtained without any restriction. To deal with a problem sometimes dubbed as "bouncing betas", we allow

$$
\frac{1}{p} \sum_{k=1}^{p} \beta_{p}=1, \beta_{k} \geq 0, k=1,2, \cdots, p
$$

Then, apply the constrained Least Squares for model (1) with constrained conditions, we can get the CLS estimator for $\beta$, and this combined forecast is given

$$
\hat{y}_{0}^{C L S}=-5.6888 * 10^{-18} f_{0}^{1}-8.7542 * 10^{-18} f_{0}^{2}-2.8844 * 10^{-16} f_{0}^{3}+0.9686 f_{0}^{4}+0.0031 f_{0}^{5}
$$

4) Variance-based, or Inverse Mean Squared Error (IMSE). This method was applied by Stock and Watson (2004) for combining forecasts of output growth. More accurate forecasting methods (lower MSE) are weighted more heavily in this method. MSE here is computed based on out-of-sample forecasts, sometimes referred to as Mean Squared Prediction Error. The IMSE combined forecast is defined as

$$
\hat{y}_{0}^{\text {IMSE }}=0.1755 f_{0}^{1}+0.1782 f_{0}^{2}+0.1771 f_{0}^{3}+0.2806 f_{0}^{4}+0.1866 f_{0}^{5}
$$

To measure the performance of the individual and combined forecasting approaches, we use the following four indexes, they are computed by the true vales and forecasting values of test set. Results are shown in Table 2 .

$$
\begin{aligned}
& M S E=\frac{1}{20} \sum_{k=1}^{20}\left(Y_{i}-\hat{Y}_{i}\right)^{2}, M A E=\frac{1}{20} \sum_{k=1}^{20}\left|Y_{i}-\hat{Y}_{i}\right|, \\
& M S P E=\frac{1}{20} \sum_{k=1}^{20}\left(\frac{Y_{i}-\hat{Y}_{i}}{Y_{i}}\right)^{2}, M A P E=\frac{1}{20} \sum_{k=1}^{20}\left|\frac{Y_{i}-\hat{Y}_{i}}{Y_{i}}\right| .
\end{aligned}
$$

From the results, it is obviously that $\mathrm{M}_{5}$ perform the worst compared with other individual forecasting model in all tables. Simple-Mean combined forecasting approache perform the worst compared with other four forecast combination methods. The proposed GWR-based combined method perform best except that its MSE is larger than that of individual model $\mathrm{M}_{3}$.

Table 2. Results of the individual and combined forecasting approaches

\begin{tabular}{ccccccccccc}
\hline & \multicolumn{4}{c}{ Single Model Forecasting } & \multicolumn{5}{c}{ Combination Forecasting Method } \\
\cline { 2 - 11 } & $M_{1}$ & $M_{2}$ & $M_{3}$ & $M_{4}$ & $M_{5}$ & SA & OLS & CLS & IMSE & GWR \\
\hline MSE & 0.0601 & 0.0610 & 0.0526 & 0.0619 & 0.0808 & 0.0610 & 0.0591 & 0.0530 & 0.0599 & 0.0565 \\
MAE & 0.1696 & 0.1705 & 0.1376 & 0.1698 & 0.1790 & 0.1607 & 0.1318 & 0.1382 & 0.1579 & 0.1295 \\
MSPE & 0.0062 & 0.0063 & 0.0051 & 0.0063 & 0.0073 & 0.0060 & 0.0052 & 0.0052 & 0.0059 & 0.0050 \\
MAPE & 0.0548 & 0.0551 & 0.0444 & 0.0547 & 0.0570 & 0.0518 & 0.0417 & 0.0446 & 0.0509 & 0.0410 \\
\hline
\end{tabular}

\section{Discussion}

To combining different individual forecasting values for one event with spatial information, this paper proposes a novel forecast combination method with varying weights. By the geographically weighted regression technique, we can obtain spatial varying weights according to the individual forecasting methods. The results on Boston house prices indicates that the procedure performs better than the other forecast combination methods.

\section{Acknowledgments}

Author Contributions: Investigation and Methodology, Chuanhua Wei; Software: Chenping Du; Writing review editing, Chuanhua Wei, Zheng Nana. All authors have read and agreed to the published version of the manuscript. 
Conflicts of Interest: The authors declare no conflict of interest.

\section{References}

Bates, J. M., \& Granger, C. W. J. (1969). The combination of forecasts. Journal of the Operational Research Society, 20(4), 451-468. https://doi.org/10.1057/jors.1969.103

Billio, M., Sartore, D., \& Toano, C. (2000). Combining forecasts: Some results on exchange and interest rates. The European Journal of Finance, 6(2), 126-145. https://doi.org/10.1080/13518470050020806

Brunsdon, C., Fotheringham, A. S., \& Charlton, M. (1996). Geographically weighted regression: A method for exploring spatial nonstationarity. Geographical Analysis, 28, 281-298. https://doi.org/10.1111/j.1538-4632.1996.tb00936.x

Chan, F., \& Pauwels, L. L. (2018). Some theoretical results on forecast combinations. International Journal of Forecasting, 34, 64-74. https://doi.org/10.1016/j.ijforecast.2017.08.005

Claeskens, G., Magnus, J. R., Vasnev, A. L., \& Wang, W. (2016). A simple theoretical explanation of the forecast combination puzzle. International Journal of Forecasting, 32(3), 754-762. https://doi.org/10.1016/j.ijforecast.2015.12.005

Deutsch, M., Granger, C. W. J., \& Terasvirta, T. (1994). The combination of forecasts using changing weights. International Journal of Forecasting, 10(1), 47-57. https://doi.org/10.1016/0169-2070(94)90049-3

Fotheringham, A. S., Brunsdon, C., \& Charlton, M. (2002). Geographically Weighted Regression the Analysis of Spatial Varying Relationships. Wiley, West Sussex.

Granger, C. W. J. (2006). Invited review combining forecast twenty years later. Journal of Forecasting, 8, 167-173. https://doi.org/10.1002/for.3980080303

Granger, C. W. J., \& Ramanathan, R. (1984). Improved methods of combining forecasts. Journal of Forecasting, 3, 197-204. http://dx.doi.org/10.1002/for.3980030207

Guidolin, M., \& Timmermann, A. (2009). Forecasts of US short-term interest rates: A flexible forecast $\begin{array}{llll}\text { combination approach. Journal of } & \text { Econometrics, }\end{array}$ https://doi.org/10.1016/j.jeconom.2008.12.004

Mojiri, A., Waghei,Y., Sani, H. R. N., \& Borzadaran, G. R. M. (2017). Comparison of Predictions by Kriging and Spatial Autoregressive Models. Communications in Statistics-Simulation and Computation, 47(6), 1785-1795. https://doi.org/10.1080/03610918.2017.1324980

Pace, R. K., \& Gilley, O. W. (1997). Using the spatial configuration of the data to improve estimation. Journal of Real Estate Finance and Economics, 14, 333-340. https://doi.org/10.1023/A:1007762613901

Raftery, A. E., Karny, M., \& Ettler, P. (2010). Online prediction under model uncertainty via dynamic model averaging: Application to a cold rolling mill. Technometrics, 52, 52-66. https://doi.org/10.1198/TECH.2009.08104

Stock, J. H., \& Watson, M. W. (2004). Combination forecasts of output growth in a seven-country data set. Journal of Forecasting, 23, 405-430. https://doi.org/10.1002/for.928

Terui, N., \& van Dijk, H. K. (2002). Combined forecasts from linear and nonlinear time series models. International Journal of Forecasting, 18, 421-438. https://doi.org/10.1016/S0169-2070(01)00120-0

Timmermann, A. (2006). Forecast combinations. In G. Elliott, C. W. J. Granger, \& A. Timmermann (Eds.), Handbook of Economic Forecasting (pp. 135-196). North-Holland, Amsterdam. https://doi.org/10.1016/S1574-0706(05)01004-9

Tsutsumi, M., \& Seya, H. (2009). Hedonic approaches based on spatial econometrics and spatial statistics: Application to evaluation of project benefits. Journal of Geographical Systems, 11, 357-380. https://doi.org/10.1007/s10109-009-0099-3

\section{Copyrights}

Copyright for this article is retained by the author(s), with first publication rights granted to the journal.

This is an open-access article distributed under the terms and conditions of the Creative Commons Attribution license (http://creativecommons.org/licenses/by/4.0/). 